\title{
The Pathogenesis and Clinical Significance of Gastrointestinal Xanthelasma: A Case Report and Review of Literature
}

\author{
Marianna G Mavilia ${ }^{1^{*}}$ and George $Y W u^{2}$ \\ ${ }^{1}$ Department of Medicine, University of Connecticut, Farmington CT, USA \\ ${ }^{2}$ Department of Gastroenterology \& Hepatology, University of Connecticut, Farmington CT, USA
}

*Corresponding author: Marianna G. Mavilia, DO, Department of Medicine, University of Connecticut, 263 Farmington Ave, Farmington CT 06030, USA, Fax: 781-545-5674

\begin{abstract}
Xanthelasmas are lesions containing lipid-filled histiocytes, which can be found on the skin or in the gastrointestinal tract. Gastrointestinal xanthelasmas (GX) are rare lesions typically found incidentally on esophagogastroduodenoscopy. Several risk factors have been identified for development of GX including dyslipidemia, fatty liver, radiotherapy, Helicobacter pylori infection, and immunosuppression. We present a case of a gastroesophageal xanthelasma in a 78-year-old male without risk factors. While his xanthelasma was anatomically located in the esophagus, the surrounding tissue was gastric mucosa, suggesting that it had occurred in a sliding hiatal hernia. The etiology of his GX was likely related to age and/ or mucosal inflammation at the hiatal hernia. Recently, studies have suggested an association of GX with malignancy. However there are no current guidelines about surveillance of GX or screening for malignancy in patients with GX. Our aim is to review a unique case of gastroesophageal GX and discuss the current literature available on this topic to highlight the clinical significance of GX.
\end{abstract}

\section{Keywords}

Xanthelasma, Gastrointestinal tract, Endoscopy, Lipid disorders, Premalignant

\section{Abbreviations}

GX: Gastrointestinal Xanthelasmas; GI: Gastrointestinal; CXs: Cutaneous Xanthelasmas; EX: Esophageal Xanthelasma; EGDs: Esophagogastroduodenoscopies

\section{Introduction}

Xanthelasmas, or xanthomas, are lesions containing lipid-rich histiocytes. They are most commonly seen on the skin but can also occur in the gastrointestinal (GI) tract. Cutaneous xanthelasmas (CXs) occur in $1.1 \%$ of females and $0.3 \%$ of males and are most commonly found in the periorbital area [1]. Gastrointestinal xanthelasmas (GXs) are extremely rare lesions with an estimated prevalence of $0.2-0.8 \%$ [2]. They have been found in various parts of the Gl tract, most commonly in the stomach with a frequency of $76 \%$ of GXs [2]. They are rarely found in the esophagus, accounting for $12 \%$ [2]. To date, there are only 22 cases of esophageal xanthelasma (EX) reported in the English literature including the case presented here [3-5]. The characteristic endoscopic appearance of GX is that of a yellow-colored nodule or polyp [6]. They are typically solitary lesions.

GX are typically asymptomatic and are discovered incidentally on esophagogastroduodenoscopies (EGDs) done for a variety of indications. However, it is unlikely that symptoms such as abdominal pain or dyspepsia, which prompted endoscopic evaluation, are caused by solitary GX lesions. In the very rare case of multiple xanthomas, or xanthomatosis, there have been few reports of GI motility disorders [7]. However, this has not been seen with solitary lesions. Here, we present a case of GX presenting as an incidental finding on $E G D$, review the literature, and discuss the clinical significance.

\section{Case Description}

A 78-year-old male with history of alcoholic cirrhosis presented to our gastroenterology clinic for evaluation of chronic normocytic anemia. A review of symptoms was positive for constipation, but negative

Citation: Mavilia MG, Wu GY (2018) The Pathogenesis and Clinical Significance of Gastrointestinal Xanthelasma: A Case Report and Review of Literature. Clin Med Rev Case Rep 5:243. doi.org/10.23937/23783656/1410243

Accepted: November 24, 2018: Published: November 26, 2018

Copyright: (C) 2018 Mavilia MG, et al. This is an open-access article distributed under the terms of the Creative Commons Attribution License, which permits unrestricted use, distribution, and reproduction in any medium, provided the original author and source are credited. 
for hematemesis, hematochezia, melena, and weight changes. An esophagogastroduodenoscopy (EGD) had been done 2 years prior and revealed portal hypertensive gastropathy with a normal esophagus and duodenum. Other past medical history included chronic obstructive pulmonary disease, hypertension, benign prostatic hypertrophy, stroke, and vascular dementia. He had no significant family or surgical history. Medications included metoprolol tartrate, lisinopril, atorvastatin, tamsulosin, finasteride, escitalopram, mirtazapine, gabapentin, and aripiprazole. His social history was

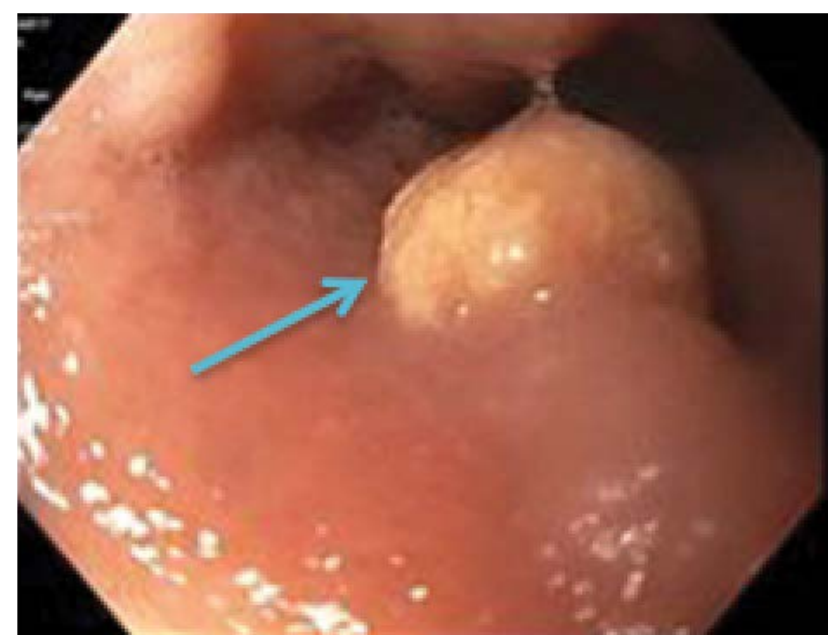

Figure 1: Endoscopic view of the distal esophageal xanthelasma showing a yellowish, polypoid appearance. significant for heavy alcohol use in the past, and a 15 pack-year smoking history.

At the time of endoscopy, his vital signs were within normal limits. He had a BMI of 22. He was an older male in no acute distress. He was normocephalic with moist mucus membranes, normal conjunctiva and clear oropharynx. His cardiopulmonary examination was normal. The abdomen was soft, nontender, and nondistended. There were no skin lesions, specifically no cutaneous xanthelasmas were noted. Recent lab work was significant for anemia with hemoglobin of 9.3 $\mathrm{g} / \mathrm{dL}$ (ref. 13.5-17.5 g/dL), but ferritin and a lipid panel was within normal limits.

He underwent EGD for variceal screening which showed grade I esophageal varices, persistent portal hypertensive gastropathy which likely contributed to his anemia. There was also a single, small, round well circumscribed, pale yellow esophageal polyp above the gastroesophageal junction, measuring $0.3 \times 0.5 \mathrm{~cm}$ (Figure 1). The lesion was biopsied due to its interval development since last EGD 2 years prior. The biopsied tissue demonstrated gastric cardia-type mucosa with abundant foam cells within the lamina propria (Figure 2). The tissue was positive for CD68, a marker of histiocytes. This was consistent with a diagnosis of xanthelasma. There was no histological evidence of metaplasia or dysplasia. Gastric biopsies were negative for Helicobacter pylori. Given the anatomic location
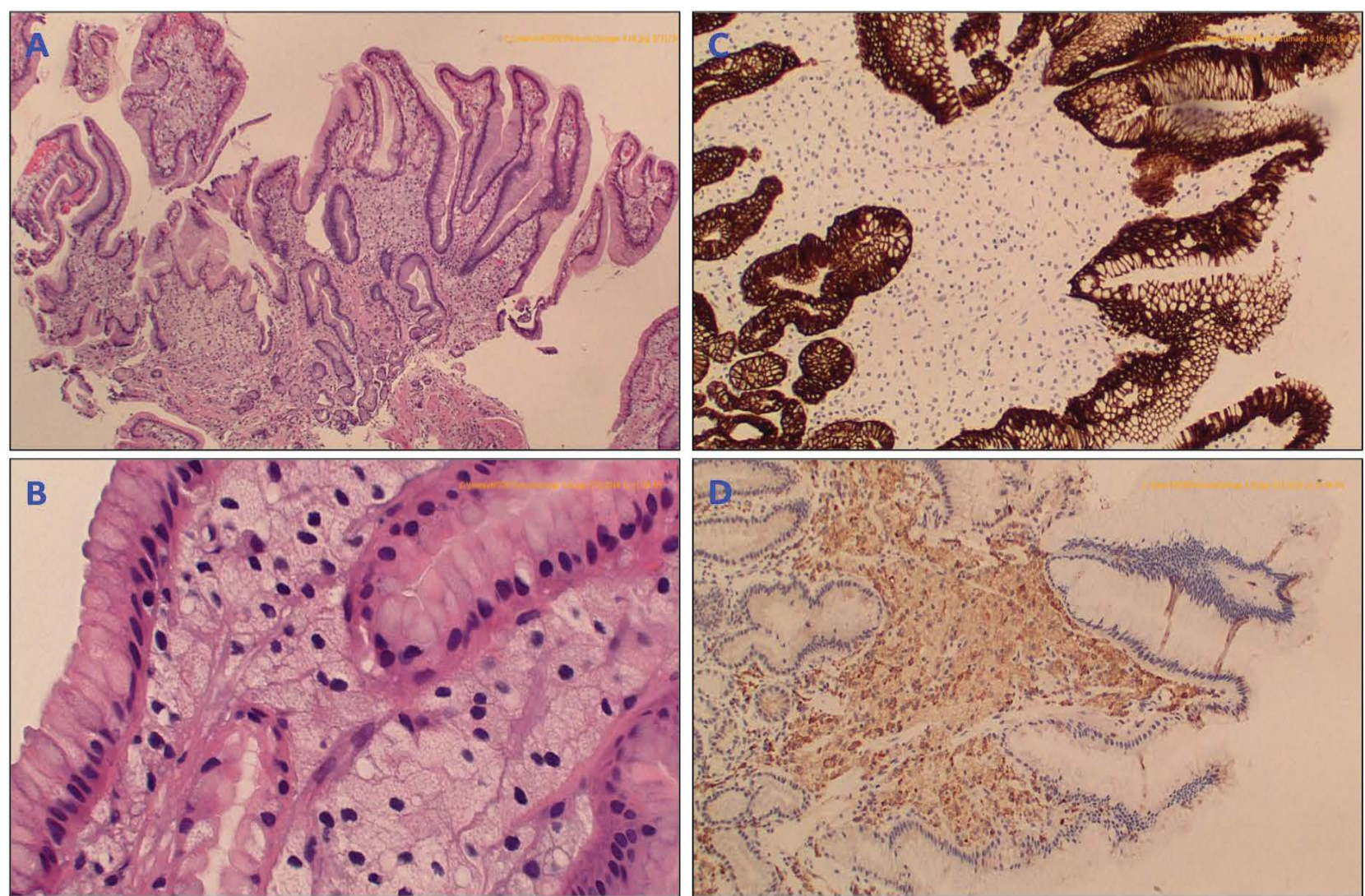

Figure 2: The biopsied tissue was prepared with H\&E staining, which is seen at $4 X(A)$ and $40 X(B)$ and demonstrates many foam cells within the lamina propria (indicated by blue arrows). Special staining was performed to differentiate the type of lesion. Staining for cytokeratin CAM (C) was negative, excluding epithelial neoplasm, however the histiocytemarker CD68 (D) was positive, confirming diagnosis of xanthelasma. 
in the esophagus but the gastric-type mucosa seen pathologically, this lesion likely originated in a segment of sliding hiatal hernia. A colonoscopy was performed at the same visit, and no colonic xanthomas were identified.

\section{Discussion and Literature Review}

\section{Risk factors}

There are several proposed risk factors for development of GX, including dyslipidemia, fatty liver, $H$. pylori infection, and immunosuppression. Our patient had no significant risk factors, making $\mathrm{GX}$ an unexpected finding in this patient.

Dyslipidemia is one of the most well-studied risk factors in the development of xanthelasmas. Patients with CX have increased LDL levels and decreased HDL levels [8]. Unlike CX, the association of GX with dyslipidemia is unclear. One study showed that the occurrence of GX was higher in patients with elevated triglyceride and fasting glucose levels compared to those with normal levels [9]. However, there was no association with hemoglobin A1c, low-density lipoprotein or total cholesterol levels [9]. This study did not describe patient characteristics such as body-mass index, waist circumference, use of cholesterol-lowering or glycemic-control medications, and other factors which may have affected the metabolic profile of their study population. Inclusion of this information would have been valuable in the interpretation of the findings relative to the general population.

A study by Chen, et al. retrospectively evaluated 1370 patients with $\mathrm{GX}$ and reported a more frequent incidence in patients with fatty liver. However, there was no relationship with chronic liver disease [9]. Unfortunately, the authors did not define which conditions were included in "chronic liver disease". Therefore, it is difficult to extrapolate this finding to our patient's alcoholic cirrhosis as a risk factor for GX.

$H$. pylori has been shown to be more prevalent in patients with $\mathrm{GX}$, at a rate of $31 \%$ [9]. The relationship between radiation and $\mathrm{GX}$ has been suggested in several case reports $[10,11]$. These observed associations support a pathogenic theory involving mucosal trauma. Radiation therapy of nearby structures may also have a role in development of GX. However, a study evaluating esophageal pathology following mediastinal radiation did not reveal any cases of EX $[10,12]$. Immunosuppression has been suggested as risk factor for GX by several case reports, however this association is not well supported in the literature $[5,13]$. Despite the numerous potential risk factors for $\mathrm{GX}$, our patient appears to have had a relatively low risk. He had no history of immunosuppression, radiation therapy, dyslipidemia, or H. pylori infection.

\section{Pathogenesis}

While the exact etiology of GX is not clear, one proposed mechanism is reactive inflammation to mucosal damage or trauma [4]. The relative frequency of these lesions along gastrointestinal tract supports this theory. The esophagus is lined with squamous epithelium which is more resistant to trauma compared to gastric columnar epithelium [7]. Therefore, the prevalence of EX is significantly less. A case report by Uehara, et al. suggested that the histiocytes involved in GX formation consist of a subtype known as M2 histiocytes [4]. Macrophages are normally found in the lamina propria of the $\mathrm{Gl}$ mucosa and remove unwanted particles using a scavenger receptor. The $\mathrm{M} 2$ histiocytes play a role in late stage inflammation, where $M 2$ cells ingest the inflammatory debris [7]. In the case of GX, histiocytes phagocytose lipids, in addition to this inflammatory debris, to form foam cells. This is consistent with the theory that GX are reactive lesions, formed in response to mucosal inflammation. Because this xanthelasma occurred within an area of hiatal hernia, mucosal inflammation due to hernia sliding, friction and reflux, likely contributed to GX development. Others have proposed that xanthelasmas develop as a normal feature of the gastrointestinal mucosa as it ages $[4,6,9,14]$. This case supports this theory as this patient developed GX at age 78 since his last endoscopic exam 2 years prior.

\section{Pathology}

At the time of this patient's EGD, the precise diagnosis of his esophageal lesion was not evident. The histologic findings delivered the final diagnosis of GX. Histologically, xanthelasmas are identified by the presence of foam cells, or histiocytes containing lipidfilled vacuoles in the lamina propria of GI mucosa or the superficial dermis layer in the skin [2]. The lipid, or oxidized low-density lipoprotein, can be distinguished with Sudan black or oil red O staining and may also help differentiate it from mucin- or glycogen- containing cells seen in Whipple's disease or other metabolic storage disorders [2]. Immunohistochemical staining is typically positive for CD68, CD163, and TGF-beta, which are markers of histiocytes [4].

\section{Clinical significance}

Although GXs are generally considered to be benign lesions, recently there have been some studies raising concern that GX may be a precursor to gastric malignancy. In a matched-control study, gastric cancer was identified in $21 \%$ of patients with a GX, compared to $3 \%$ in patients without [1]. The authors concluded that GX was significantly associated with gastric cancer and GX may represent a "biomarker" for malignancy [1]. Additionally, gastric cancer was noted to be more common in patients with 3 or more GXs [6]. In that study, the prevalence of GX was $7.7 \%$, which is significantly 
higher than the prevalence of the general population. Therefore, there may have been some population bias. Another study demonstrated a higher rate of intestinal metaplasia and dysplasia in patients with GX [9]. More specifically, intestinal metaplasia was seen in $49.6 \%$ of patients with $\mathrm{GX}$ and dysplasia was seen in $2.1 \%$, which was 7-times greater than in patients without GX [9]. A case report also suggested a link between $G X$ and malignancy, and proposed that underlying cancer cells may stimulate proliferation of xanthoma cells [15]. Due to the high mortality of gastric cancer, it is important to determine whether GXs are a precursor lesion of gastric cancer. The current data represent only statistical associations, and there is currently no clear link to any other GI malignancy. Additionally, it is unclear if there is a malignant potential for xanthelasmas in other parts of the gastrointestinal tract, such as the esophagus.

\section{Conclusion}

Our case highlights the pathogenesis of GX. Arising within a hiatal hernia, mucosal injury from sliding friction and reflux predisposed this patient to development of a $G X$. This case also raised the question about surveillance of these types of lesions given the possible risk for premalignancy. Currently, there are no guidelines regarding surveillance for of GX or screening for GI malignancy in patients with GX. While this patient may need further endoscopic evaluation for other reasons, we felt there was no need for additional endoscopic follow up for his GX. Overall, the potential of GX to "predict" gastrointestinal cancer requires further investigation.

\section{Acknowledgements}

Special thanks to Dr. Poormina Hegde of the University of Connecticut Department of Pathology for providing images and descriptions of pathologic components of this case.

\section{Financial Support}

None.

\section{Author Contributions}

Mavilia: Drafting manuscript, literature review; Wu: Clinical supervision of case, major revisions.

\section{References}

1. Kavoussi H, Ebrahimi A, Rezaei M, Najafi B, Kavoussi R, et al. (2016) Serum lipid profile and clinical characteristic of patients with xanthelasma palpebrum. An Bras Dermatol 91: 468-471.

2. Gencosmanoglu R, Sen-Oran E, Kurtkaya-Yapicier O, Tozun N (2005) Xanthelasmas of the upper gastrointestinal tract. J Gastroenterol 40: 657.

3. Dirweesh A, Khan M, Bukhari S, RImmer C, Shmuts R (2017) Nodular Esophageal Xanthoma: A Case Report and Review of the Literature. Case Rep Gastrointest Med 2017.

4. Uehara K, Iwashita $\mathrm{H}$, Tanabe $\mathrm{Y}$, Kurima K, Oshiro M, et al. (2017) Esophageal Xanthoma: Presence of M2 Macrophages Suggests Association with Late Inflammatory and Reparative Processes. Open Med (Wars) 12: 335-339.

5. Diaz Del Arco C, Alvarez Sanchez A, Fernandez Acenero MJ (2016) Non-gastric gastrointestinal xanthomas: Case series and literature review. J Gastrointestin Liver Dis 25: 389-394.

6. Sekikawa A, Fukui H, Maruo T, Tsumura T, Kanesaka T, et al. (2014) Gastric xanthelasma may be a warning sign for the presence of early gastric cancer. J Gastroenterol Hepatol 19: 951-956.

7. Sagaert X, Tousseyn T, De Hertogh G, Geboes K (2012) Macrophage-related diseases of the gut: A pathologist's perspective. Virchows Arch 460: 555-567.

8. Ribera M, Pinto X, Argimon JM, Fiol C, Puiol R, et al. (1995) Lipid metabolism and apolipoprotein E phenotypes in patients with xanthelasma. Am J Med 99: 485-490.

9. Chen Y, He XJ, Zhou MJ, Li YM (2017) Gastric xanthelasma and metabolic disorders: A large retrospective study among Chinese population. Work J Gastroenterol 23: 7756-7764.

10. Salamanca J, Alemany I, Sosa G, Pinedo F, Hernando S, et al. (2012) Esophageal verruciform xanthoma following radiotherapy. Gastroenterol Hepatol 35: 317-320.

11. Herrera-Goepfert R, Lizano-Soberon M, Garcia-Perales M (2003) Verruciform xanthoma of the esophagus. Hum Pathol 34: 814-815.

12. Farwell DG, Rees CJ, Mouadeb DA, Allen J, Chen AM, et al. (2010) Esophageal pathology in patients after treatment for head and neck cancer. Otolaryngol Head Neck Surg 143: 375-378.

13. Barrera-Herrara LE, Arias F, Rodriguez-Urrego PA, PalauLazaro A (2015) Small Bowel obstruction due to Intestinal Xanthomatosis. Case Rep Pathol 2015: 231830.

14. Dhakal M, Dhakal OP, Bhandari D, Gupta A (2013) Gastric xanthelasma: an unusual endoscopic finding. BMJ Case Rep 2013.

15. Muraoka A, Suehiro I, Fuji M, Uneo H, Hayashi S, et al. (1998) Type Ila early gastric cancer with proliferation of xanthoma cells. J Gastroenterol 33: 326-329. 